\title{
Comparison Of Chemical And Mineral Content Of Milk From Human, Cow, Buffalo, Camel And Goat In Egypt.
}

\author{
Ghada Z A Soliman \\ Lecturer of Biochemistry, Biochemistry Department, National Nutrition Institute, Cairo
}

\begin{abstract}
Milk is an important food; it is considered as a good source of Ca. Comparison studies of chemical and mineral contents of milk from human, cow, buffalo, camel and goat in Egypt are rare, so we determine chemical and mineral composition cows; camels; buffalo and goats milk in Egypt and to compare with the human milk since such comparisons are rare in Egypt. There are several studies dealing with the chemical composition of milk, but they are foreign, nonEgyptian. It is known that changing of environment has a significant effect on natural physiological function of both human and animals, so it was very important to made such study on Egyptian environment (ecology), hoping to give understanding and explain some of the malnutrition problems in Egypt. Three hundred and two milk samples were obtained at random from: cows; camels; buffalo and goats (n: 44, 108, 40, 40 respectively), while human milk was

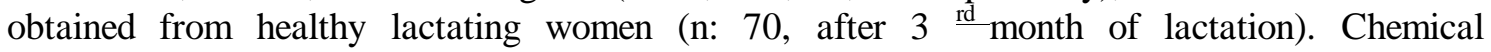
constituents as protein, fat, carbohydrates, moisture, ash and lactose were determined. Mineral contents as calcium $(\mathrm{Ca})$, iron $(\mathrm{Fe})$, Zinc $(\mathrm{Zn})$, sodium $(\mathrm{Na})$, potassium $(\mathrm{K})$, magnesium $(\mathrm{Mg})$, copper $(\mathrm{Cu})$, phosphorous $(\mathrm{P})$ and manganese $(\mathrm{Mn})$ were measured in the ash. Human milk contains the lowest protein and ash content. Buffalo milk has the highest fat and total solids content. It gives the higher yield of energy. Human milk contains the highest carbohydrates and lactose content (4.98 gm\% carbohydrates, of which 4.70 are lactose, i.e. it represent $94.46 \%$ ). Human milk contains the lowest concentration of $\mathrm{Ca}, \mathrm{P}, \mathrm{Fe}, \mathrm{Zn}, \mathrm{Na}, \mathrm{K}$ and $\mathrm{Mg}$. Ca: $\mathrm{P}$ ratio of human milk is higher (2.32 vs 1.18-1.47) than other species. Mn content of human milk is higher (human milk contains $\approx 6.5$ times camel and cow milk and 24.14 times as goat milk). Buffalo milk has the highest $\mathrm{Ca}, \mathrm{P}$ and $\mathrm{Mg}$ content. Camel milk has the highest $\mathrm{Fe}, \mathrm{Zn}, \mathrm{Na}$ and $\mathrm{Cu}$ content. Goat milk has the highest $\mathrm{K}$ content. Chemical and mineral content of the 5 studied species varied widely. Camel, buffalo, cow and goat milk cannot replace human milk but it may complement it.
\end{abstract}

Key words: goat, cow, human, camel, buffalo, mineral composition, chemical composition.

\section{Introduction}

Milk is an excellent source of most essential minerals for human. It contains mostly calcium, phosphorus and constitutes the most important source of bioavailable calcium in our diet (ICAR, 1981). Milk and dairy products are part of a healthy diet. The composition of the milk of various animal species differs, but in every case it has a high priority in human nutrition.

More than 5\% of the world's milk comes from buffaloes. Over $95 \%$ of the world buffalo milk is produced in Asia (Charan, 1994). Buffalo milk is used in much the same way as cow's milk. It is high in fat and total solids, which gives it a rich flavor. Many people prefer it than cow's milk. In Egypt, for example, the severe mortality rate among buffalo calves is due in part to the sale of buffalo milk, which is in high demand, thus depriving calves of proper nourishment. The buffaloes of Egypt are used mainly for milk production. Buffalo milk is pure white because it contains no carotene since buffalo have already processed the carotene into vitamin A. Buffalo milk is also very thick.

The value of goat milk in human nutrition has so far received very little 


\section{Ghada Z A Soliman}

factual and academic attention (Haenlein 1992; Park, 1991) despite its medical need for some people especially infants afflicted with various ailments, including cow milk protein sensitivities (Lothe et al., 1982 and Host et al., 1988). Goat milk proteins and fats have many significant differences in their compositions from the milk of other mammalian species, especially in relative proportions of the various milk proteins and fats and in their genetic polymorphisms (Ambrosoli et al., 1988). Goat milk have shorter rennet coagulation time, less resistance to heat treatment, curd firmness is weaker and cheese yields are less which might explain significant differences to cow and other milk in digestion by infants and patients which traditionally have been explained by the "homogenized" nature of goat milk fat (Haenlein 1992; Park, 1991).

Human milk is believed to provide all the nutrients and essential minerals and trace elements (micronutrients) that are required by the normal term infant growth, until weaning. With a few exceptions, excessive micronutrient supplies to the mother, or a moderate deficiency in her diet, do not greatly alter the supply to the infant. Thus, the infant is well protected by maternal homeostatic processes (Bates and Prentice., 1994). There is a wide variation in the reported data on the concentrations of trace elements in human milk from different countries. The composition of human milk varies over the course of lactation and in each individual. Human milk is markedly different from cows' milk, both in terms of macronutrients and micronutrients.

It is noteworthy that milk of each species has a particular individual pattern of minerals, which may be a pointer ofrelative nutritional importance of the element.

The aimof this work was to investigate and compare the mineral composition of human, cow, buffalo, camel and goat milk in Egypt since such comparisons are rare in Egypt. There are several studies dealing with the chemical composition of milk, but they are foreign, non- Egyptian. It is known that changing of environment has a significant effect on antural physiological function of both human and animals, so it was very important to made such study on
Egyptian environment (ecology), hoping to give understanding and explain some of the malnutrition problems in Egypt.

\section{Materials And Methods Sampling}

Milk samples were obtained from: cows; camels; buffalo and goats (n: 44, 108, 40, 40 respectively), while human milk was obtained from healthy lactating women (n: 70 , after 3 rd month of lactation to get mature milk). Milk samples were collected and stored in ice tank until reaching the laboratory, then frozen at $4{ }^{\circ} \mathrm{C}$ if to be analyzed in $2-3$ days or at $20^{\circ} \mathrm{C}$ if to be analyzed in few days if not analyzed immediately. Interestingly, camels' milk did not sour at $4^{\circ} \mathrm{C}$ for up to 2 months as the remaining milk, which agree with Yagil et al. (1984).

\section{Chemical Analysis}

Chemical constituents as protein, fat, carbohydrates, moisture, ash and lactose were determined according to AOAC (1990). Mineral contents as calcium (Ca), iron $(\mathrm{Fe})$, Zinc $(\mathrm{Zn})$, sodium $(\mathrm{Na})$, potassium $(\mathrm{K})$, magnesium $(\mathrm{Mg})$, copper $(\mathrm{Cu})$ and manganese $(\mathrm{Mn})$ were measured in the ash using atomic absorption, Unicam 929 (AOAC, 1981). Phosphorous (P) was estimated colorimetrically in the ash according to $(\mathrm{AOAC}, 1981)$ and $\mathrm{Ca} / \mathrm{P}$ was calculated.

All data collected were subjected to a completely randomized design model and covariance analysis (Steel and Torrie 1969).

\section{Results}

Tables (1-3) showed the chemical composition of camels', human's, goat's, buffalo's and cows' milk, and the significant differences between and among them. Human milk contained (g/100 g): 1.11 protein; 4.17 fat; 0.21 ash; 86.47 water; 13.53 total solids; 8.04 carbohydrates, of which 7.12 are lactose (88.57\%).Human milk contain significantly less protein content than the remaining camel, goat, cow and buffalo milk where they have a somewhat similar content (1.11 vs 3.27, 
4.02, 3.48 and $3.32 \mathrm{~g} \%$ respectively). Content of human milk fat is close to goat, cow and camel (4.17 vs 4.04-4.2). Content of human milk total solids is significantly higher than goat and camel and comparable with cow milk (13.53 vs $12.62-12.95$, S; vs 13.3, NS, respectively). Human milk contains significantly higher carbohydrates and lactose content than goat, camel, cow and buffalo (8.04 vs $4.44-5.33,+61.47 \%$ $+81.17 \% ; 7.12$ vs $4.27-5.02,+51.4-$ $+66.75 \%$ respectively). Human milk gives significantly higher calories $(74.13 \mathrm{Kcal})$ than camel, cow and goat milk $(+4.32-$ $+10.05 \%)$ and significantly lower calories than buffalo milk (-29.47\%), tables (2-3).

Buffalo milk contained (g/100 g): 4.02 protein; 7.52 fat; 0.80 ash; 82.33 water; 17.65 total solids; 5.33 carbohydrates, of which 5.02 are lactose $(94.32 \%)$. Buffalo milk contained less water, more total solids, more fat $(7.52 \pm 0.07$ vs $4.14 \pm 0.09,+81.0 \%)$, more lactose, and more protein than cow's milk.

Goat milk contained (g/100 g): 3.32 protein; 4.04 fat; 0.83 ash; 87.38 water; 12.62 total solids; 4.44 carbohydrates, of which 4.27 are lactose $(96.26 \%)$. The main component of goat milk as protein, total solids, carbohydrate and lactose differs from cow milk. It has significantly lower content while fat content is somewhat similar.

Camel milk contained $(\mathrm{g} / 100 \mathrm{~g}): 3.27$ protein; 4.20 fat; 0.75 ash; 87.05 water; 12.95 total solids; 4.67 carbohydrates, of which 4.31 are lactose $(92.37 \%)$. Camel milk is significantly different from buffalo and human milk. Camel milk has highly significant protein and mineral content than human milk but lower than buffalo milk. It has a similar fat content as human but significantly lower than buffalo milk. Camel milk resembles goat milk than cow milk. It has a comparable content of fat, moisture, total solids and carbohydrate with cow and goat milk. Camel milk has similar protein and lactose content as goat milk but significantly lower protein and lactose than cow milk.

Cow milk contained (g/100 g): 3.48 protein; 4.14 fat; 0.71 ash; 86.70 water; 13.30 total solids; 4.98 carbohydrates, of which 4.70 are lactose (94.46\%). Cow milk is significantly different from buffalo, goat, camel and human milk. Cow milk has highly significant protein content than human, camel and goat milk but lower than buffalo milk. Cow milk has significantly lower fat content than buffalo but similar to the other milks. It also has significantly lower ash content than the remaining milk except human milk where it has significantly higher ash content (table 2-3). In general, human milk contained the lowest protein content while buffalo milk contained the highest content $(1.11 \pm 0.04$ vs $4.02 \pm 0.05)$. Goat milk contained the lowest fat content while buffalo milk contained the highest content $(4.04 \pm 0.05$ vs $7.52 \pm 0.07)$. Buffalo milk contained the lowest water content while goat milk contained the highest content $(82.33 \pm 0.10 \quad$ vs $87.38 \pm 0.06$ ). Goat milk contained the lowest total solids content and lowest total energy while buffalo milk contained the highest content of them $(12.62 \pm 0.06$ vs $17.65 \pm 0.10 ; 67.36 \pm 0.84$ vs $105.11 \pm 1.21$ respectively). Human milk contained the highest lactose content $(7.12 \pm 0.07)$ where it comprises $96.26 \%$ of the total carbohydrate.

Table (4) showed the different contribution of camel, buffalo, cow, goat and human milk intake with the recommended human daily dietary allowances, RDA, 2002. A cup (250 gm) of human milk provides infants $\leq 1 \mathrm{y}$ with $\approx 25.0-30.0 \%$ of their RDA of protein vs $89.82-110.37 \%$ of other milks; $\approx 21.0-33 \%$ of their RDA of carbohydrate vs $12-22 \%$ of other milks; and $\approx 34.0 \%$ of their RDA (or DRI) of fat vs $33-63 \%$ of other milks. It agrees with Whitehead (1995).

Camels', women's, goat's, buffalo's and cows' milk were kept freezed if it is not analyzed immediately and needs to be kept for a few days.

Tables (6-8) show elements content $(\mathrm{mg} / 100 \mathrm{~g})$ of Egyptian human, cow, buffalo, camel and goat milk and the significant differences between and among them were shown. The data of the table 1 indicate that cow, buffalo, camel and goat milk contain 3.41-3.99 times as human milk which did not agree with ICAR (1981). 


\section{Ghada Z A Soliman}

ICAR (1981) had reported that goat milk had 7-10 times minerals as human and cow milk. In this study cow, buffalo, camel and goat milk had highest mineral contents compared to that of human milk with significant differences between them.

Calcium (Ca): Data of table (6) shows calcium content of camel, buffalo, cow, goat and human. In this study buffalo then goat milk provide a great amount of calcium.

Phosphorous: Data of table (6) shows phosphorous content of camel, buffalo, cow, goat and human. Human milk had significantly lower content while camel had significantly higher levels. Buffalo and goat had somewhat comparable levels.

The Calcium: Phosphorus ratio (Ca: P): A significant difference was found among all tested milk types especially against human milk where a significant decrease was found (from $-36.59 \%$, buffalo, to $-48.95 \%$, goat, tables 2-3).

Iron $(\mathrm{Fe})$ : Data of table (6) shows iron content of camel, buffalo, cow, goat and human. There is a significant difference (decrease) between: camel, buffalo milk with cow, goat and human milk. There is a significant increase between camel and buffalo milk. There is no significant difference in the iron content of cow and goat or cow with human milk; or goat and human milk. Camel had the highest iron concentration then buffalo. While cow, goat and human have a comparable concentration.

Zinc (Zn): Data of table (6) shows Zinc content of camel, buffalo, cow, goat and human. Human milk had significantly lower content while camel had significantly higher levels. Cow and goat had somewhat comparable levels.

Sodium (Na): Data of table (6) shows sodium content of camel, buffalo, cow, goat and human. Camel milk had significantly higher $\mathrm{Na}$ content while human had the lowest content. Buffalo, cow and goat milk had non significantly comparable levels of $\mathrm{Na}$.

Potassium (K): Data of table (6) shows potassium content of camel, buffalo, cow, goat and human was. The Potassium content of goat milk was significantly higher than the remaining milk, while human milk contained significantly lower content.

Magnesium (Mg): Data of table (6) shows magnesium content of camel, buffalo, cow, goat and human was. The Magnesium content of buffalo milk was significantly higher than the remaining milk. The magnesium content of cow and goat milk was similar.

Copper $(\mathrm{Cu})$ : Data of table (6) show copper content of camel, buffalo, cow, goat and human. The Copper content of camel milk was significantly higher than the remaining milk. The copper content of buffalo and goat milk was similar.

Manganese (Mn): Data of table (6) shows copper content of camel, buffalo, cow, goat and human. Human milk contained the highest significant content of $\mathrm{Mn}$, while cow milk contained the lowest content. Camel milk content is comparable with goat milk (no significant difference).

Table (1): Chemical composition of Egyptian human, cow, buffalo, camel and goat milk $(\mathrm{g} / \mathbf{1 0 0 g})$. Results are expressed as Mean $\pm \mathrm{SE}$

\begin{tabular}{|l|l|l|l|l|l|l|l|l|l|}
\hline & Protein & Fat & Ash & Moisture & Total Solids & $\begin{array}{l}\text { larbo- } \\
\text { hydrate }\end{array}$ & $\begin{array}{l}\text { Total } \\
\text { Energy }\end{array}$ & Lactose & $\%$ Lactose \\
\hline 1- Camel & $3.27 \pm 0.06$ & $4.2 \pm 0.11$ & $0.75 \pm 0.01$ & $87.05 \pm 0.13$ & $12.95 \pm 0.13$ & $4.67 \pm 0.10$ & $70.09 \pm 0.91$ & $4.31 \pm 0.10$ & $92.37 \pm 0.53$ \\
\hline 2- Buffalo & $4.02 \pm 0.05$ & $7.52 \pm 0.07$ & $0.80 \pm 0.00$ & $82.33 \pm 0.10$ & $17.65 \pm 0.10$ & $5.33 \pm 0.05$ & $105.11 \pm 1.21$ & $5.02 \pm 0.03$ & $94.32 \pm 0.32$ \\
\hline 3- Cow & $3.48 \pm 0.03$ & $4.14 \pm 0.09$ & $0.71 \pm 0.00$ & $86.70 \pm 0.13$ & $13.30 \pm 0.13$ & $4.98 \pm 0.02$ & $71.06 \pm 1.05$ & $4.70 \pm 0.02$ & $94.46 \pm 0.23$ \\
\hline 4- Goat & $3.32 \pm 0.03$ & $4.04 \pm 0.05$ & $0.83 \pm 0.00$ & $87.38 \pm 0.06$ & $12.62 \pm 0.06$ & $4.44 \pm 0.02$ & $67.36 \pm 0.84$ & $4.27 \pm 0.02$ & $96.26 \pm 0.13$ \\
\hline 5- Human & $1.11 \pm 0.04$ & $4.17 \pm 0.06$ & $0.21 \pm 0.003$ & $86.47 \pm 0.08$ & $13.53 \pm 0.08$ & $8.04 \pm 0.06$ & $74.13 \pm 0.57$ & $7.12 \pm 0.07$ & $88.57 \pm 0.54$ \\
\hline
\end{tabular}


Table (2): Significant differences of chemical contents of milk between different species

\begin{tabular}{|c|c|c|c|c|c|c|c|c|c|}
\hline & Protein & Fat & Ash & Moisture & \begin{tabular}{|l} 
Total \\
Solids \\
\end{tabular} & $\begin{array}{l}\text { Carbo- } \\
\text { hydrate }\end{array}$ & $\begin{array}{l}\text { Total } \\
\text { Energy }\end{array}$ & Lactose & $\begin{array}{l}\% \\
\text { Lactose }\end{array}$ \\
\hline Camel vs Buffalo & 0.0001 & 0.0001 & 0.0002 & 0.0001 & 0.0001 & 0.0005 & 0.0001 & 0.0002 & 0.04 \\
\hline Camel vs Cow & 0.03 & NS & 0.001 & NS & NS & NS & NS & 0.02 & 0.02 \\
\hline Camel vs Goat & NS & NS & 0.001 & NS & NS & NS & NS & NS & 0.0001 \\
\hline Camel vs Human & 0.0001 & NS & 0.0001 & 0.001 & 0.001 & 0.0001 & 0.001 & 0.0001 & 0.0001 \\
\hline Buffalo vs Cow & 0.0001 & 0.0001 & 0.0001 & 0.0001 & 0.0001 & 0.0004 & 0.0001 & 0.0001 & NS \\
\hline Buffalo vs Goat & 0.0001 & 0.0001 & 0.0001 & 0.0001 & 0.0001 & 0.0001 & 0.0001 & 0.0001 & 0.002 \\
\hline Buffalo vs Human & 0.0001 & 0.0001 & 0.0001 & 0.0001 & 0.0001 & 0.0001 & 0.0001 & 0.0001 & 0.0001 \\
\hline Cow vs Goat & 0.046 & NS & 0.0001 & 0.006 & 0.006 & 0.001 & 0.04 & 0.0001 & 0.0002 \\
\hline Cow vs Human & 0.0001 & NS & 0.0001 & NS & NS & 0.0001 & 0.03 & 0.0001 & 0.0001 \\
\hline Goat vs Human & 0.0001 & NS & 0.0001 & 0.0001 & 0.0001 & 0.0001 & 0.0001 & 0.0001 & 0.0001 \\
\hline
\end{tabular}

Table (3): \% change of chemical contents of milk between and among different species.

\begin{tabular}{|l||l|l|l|l|l|l|l|l|l|l||}
\hline & Protein & Fat & Ash & Moisture & $\begin{array}{l}\text { Total } \\
\text { Solids }\end{array}$ & $\begin{array}{l}\text { Carbo- } \\
\text { hydrate }\end{array}$ & $\begin{array}{l}\text { Total } \\
\text { Energy }\end{array}$ & Lactose & $\begin{array}{l}\% \\
\text { Lactose }\end{array}$ \\
\hline \hline Camel & $22.87^{*}$ & $76.57^{*}$ & $5.71^{*}$ & $-5.42^{*}$ & $36.46^{*}$ & $14.31^{*}$ & $49.97^{*}$ & $16.44^{*}$ & $2.11^{*}$ \\
\hline Buffalo vs Camel & $6.38^{*}$ & -2.89 & $-5.89^{*}$ & -0.41 & 2.72 & 6.67 & 1.39 & $9.03^{*}$ & $2.27^{*}$ \\
\hline Cow vs Camel & 1.66 & -5.28 & $10.10^{*}$ & 0.37 & -2.51 & -4.93 & -3.89 & -1.01 & $4.21^{*}$ \\
\hline Goat vs Camel & $-65.99^{*}$ & -2.11 & $-72.38^{*}$ & $-0.67^{*}$ & $4.47^{*}$ & $72.24^{*}$ & $5.77^{*}$ & $65.08^{*}$ & $-4.11^{*}$ \\
\hline Human vs Camel & $-18.62^{*}$ & $-43.36^{*}$ & $-5.40^{*}$ & $5.73^{*}$ & $-26.72^{*}$ & $-12.51^{*}$ & $-33.32^{*}$ & $-14.12^{*}$ & $-2.07^{*}$ \\
\hline Buffalo & $-13.42^{*}$ & $-45.00^{*}-10.98^{*}$ & $5.31^{*}$ & $-24.72^{*}$ & $-6.68^{*}$ & $-32.39^{*}$ & $-6.37^{*}$ & 0.15 \\
\hline Camel vs Buffalo & $-17.26^{*}$ & $-46.35^{*}$ & $4.15^{*}$ & $6.13^{*}$ & $-28.55^{*}$ & $-16.83^{*}$ & $-35.91^{*}$ & $-14.98^{*}$ & $2.06^{*}$ \\
\hline Cow vs Buffalo & $-72.32^{*}$ & $-44.56^{*}-73.88^{*}$ & $5.03^{*}$ & $-23.44^{*}$ & $50.69^{*}$ & $-29.47^{*}$ & $41.77^{*}$ & $-6.10^{*}$ \\
\hline Goat vs Buffalo \\
\hline Human vs Buffalo \\
\hline Cow
\end{tabular}


Table (4): \% contribution of camel, buffalo, cow, goat and human milk intake with the recommended human daily dietary allowances, RDA (2002)

\begin{tabular}{|c|c|c|c|c|c|c|c|c|c|c|}
\hline & Protein & Fat & $\begin{array}{l}\text { Carbo- } \\
\text { hydrate }\end{array}$ & Protein & Fat & $\begin{array}{l}\text { Carbo- } \\
\text { hydrate }\end{array}$ & Protein & $\begin{array}{l}\text { Carbo- } \\
\text { hydrate }\end{array}$ & Protein & $\begin{array}{l}\text { Carbo- } \\
\text { hydrate }\end{array}$ \\
\hline & \multicolumn{6}{|l|}{ Children } & \multicolumn{4}{|l|}{ Adult } \\
\hline & \multicolumn{3}{|c|}{$0-6 m$} & \multicolumn{3}{|c|}{$7-12 \mathrm{~m}$} & \multicolumn{2}{|l|}{ Male } & \multicolumn{2}{|l|}{ Female } \\
\hline Camel & 89.82 & 34.36 & 19.44 & 74.31 & 35.50 & 12.28 & 14.60 & 8.97 & 17.77 & 8.97 \\
\hline Buffalo & 110.37 & 60.67 & 22.23 & 91.31 & 62.69 & 14.04 & 17.94 & 10.26 & 21.83 & 10.26 \\
\hline \begin{tabular}{|l|} 
Cow \\
\end{tabular} & 95.55 & 33.37 & 20.74 & 79.05 & 34.48 & 13.10 & 15.53 & 9.57 & 18.90 & 9.57 \\
\hline \begin{tabular}{|l|} 
Goat \\
\end{tabular} & 91.32 & 32.54 & 18.49 & 75.55 & 33.63 & 11.68 & 14.84 & 8.53 & 18.07 & 8.53 \\
\hline Human & 30.55 & 33.63 & 33.49 & 25.27 & 34.75 & 21.15 & -- & - & -- & -- \\
\hline
\end{tabular}

Note: This figures is per serving cup (=250 gm)

Table (5): Comparison of chemical milk constituents with other reported contents.

\begin{tabular}{|c|c|c|c|c|c|c|c|c|c|c|}
\hline & & Protein & Fat & Ash & Moisture & \begin{tabular}{|l} 
Total \\
Solids \\
\end{tabular} & $\begin{array}{l}\text { Carbo- } \\
\text { hydrate }\end{array}$ & $\begin{array}{l}\text { Total } \\
\text { Energy }\end{array}$ & Lactose & $\begin{array}{l}\% \\
\text { Lactose }\end{array}$ \\
\hline \multirow[t]{3}{*}{ Camel } & $*$ & 3.27 & 4.26 & 0.75 & 87.05 & 12.95 & 4.67 & 70.1 & 4.31 & 92.37 \\
\hline & $\mid$\begin{tabular}{||l|l|} 
\\
\end{tabular} & 3.5 & 3.8 & 0.8 & 87.9 & 12.1 & 4 & 64.2 & 3.9 & 97.5 \\
\hline & 2 & 4.5 & 5.5 & 0.9 & 85.6 & 14.4 & 3.5 & 81.5 & 3.4 & 97.14 \\
\hline \multirow[t]{3}{*}{ Buffalo } & $*$ & 4.02 & 7.52 & 0.8 & 82.33 & 17.65 & 5.33 & 105.11 & 5.02 & 94.32 \\
\hline & 3 & 3.8 & 7.4 & 0.8 & 83.17 & 16.83 & 4.83 & 101.12 & 4.8 & 99.38 \\
\hline & 4 & 4.16 & 7.96 & 0.78 & 82.09 & 17.91 & 5.01 & 108.32 & 4.86 & 97.006 \\
\hline \multirow[t]{3}{*}{ Cow } & $*$ & 3.48 & 4.14 & 0.71 & 86.7 & 13.3 & 4.98 & 71.06 & 4.7 & 94.46 \\
\hline & 3 & 3.4 & 3.5 & 0.7 & 87.8 & 12.2 & 4.6 & 63.5 & & \\
\hline & 4 & 3.58 & 4.14 & 0.71 & 86.43 & 13.57 & 5.14 & 72.14 & 4.96 & 96.5 \\
\hline \multirow[t]{2}{*}{ Goat } & $*$ & 3.32 & 4.04 & 0.83 & 87.38 & 12.62 & 4.44 & 67.36 & 4.27 & 96.26 \\
\hline & $6 \& 7$ & 3.56 & 4.14 & 0.82 & 87.03 & 12.97 & 4.45 & 69.3 & & \\
\hline \multirow[t]{3}{*}{ Human } & $*$ & 1.11 & 4.17 & 0.21 & 86.47 & 13.53 & 8.04 & 74.13 & 7.12 & 88.57 \\
\hline & 8 & 1.1 & 4.5 & 0.2 & 87.1 & 12.9 & 7.1 & 73.3 & 6.8 & 95.77 \\
\hline & 5 & 0.9 & 3.2 & 0.21 & 88.29 & 11.71 & 7.4 & 63 & & \\
\hline
\end{tabular}

* Present study, ${ }^{1}$ El-Bahay, $1962,{ }^{2}$ Knoess, 1976 and1977; ${ }^{3}$ Wong et al. $1988 ;{ }^{4}$ Byron et al. 2005; ${ }^{5}$ Renner, 1983, 1989; ${ }^{6}$ Posati and Orr, 1976; ${ }^{7}$ Alichanidis and Polychroniadou, 1996; ${ }^{8}$ Fomon, 1974, 1993.

Table (6): Mineral composition of Egyptian human, cow, buffalo, camel and goat milk $(\mathrm{mg} / \mathbf{1 0 0 g})$. Results are expressed as Mean $\pm \mathrm{SE}$

\begin{tabular}{|l|l|l|l|l|l|l|l|l|l|l|l||}
\hline & & $\mathrm{Ca}$ & $\mathrm{P}$ & $\mathrm{Ca} / \mathrm{P}$ & $\mathrm{Fe}$ & $\mathrm{Zn}$ & $\mathrm{Na}$ & $\mathrm{K}$ & $\mathrm{Mg}$ & $\mathrm{Cu}$ & $\mathrm{Mn}$ \\
\hline I-Camel & Mean \pm & $111.36 \pm$ & $81.17 \pm$ & $1.37 \pm$ & $0.23 \pm$ & $0.51 \pm$ & $57.84 \pm$ & $156.32 \pm$ & $6.70 \pm$ & $0.061 \pm$ & $0.013 \pm$ \\
& SE & 4.36 & 3.08 & 0.01 & 0.01 & 0.015 & 1.22 & 2.85 & 0.14 & 0.0023 & 0.0006 \\
\hline \multirow{2}{*}{ II-Buffalo } & Mean \pm & $163.19 \pm$ & $111.36 \pm$ & $1.47 \pm$ & $0.135 \pm$ & $0.24 \pm$ & $51.61 \pm$ & $167.18 \pm$ & $29.56 \pm$ & $0.04 \pm$ & $0.01833 \pm$ \\
& SE & 4.56 & 2.61 & 0.04 & 0.007 & 0.008 & 0.66 & 3.16 & 0.79 & 0.0025 & 0.00034 \\
\hline \multirow{2}{*}{ III-Cow } & Mean \pm & $119.90 \pm$ & $95.03 \pm$ & $1.26 \pm$ & $0.07 \pm$ & $0.38 \pm$ & $49.67 \pm$ & $147.02 \pm$ & $13.42 \pm$ & $0.017 \pm$ & $0.0037 \pm$ \\
& SE & 0.69 & 0.72 & 0.01 & 0.02 & 0.00 & 0.70 & 1.55 & 0.24 & 0.0016 & 0.0001 \\
\hline IV-Goat & Mean \pm & $130.28 \pm$ & $110.16 \pm$ & $1.18 \pm$ & $0.06 \pm$ & $0.32 \pm$ & $50.33 \pm$ & $201.45 \pm$ & $13.87 \pm$ & $0.04 \pm$ & $0.0138 \pm$ \\
& SE & 2.26 & 1.61 & 0.01 & 0.00 & 0.03 & 0.77 & 1.90 & 0.11 & 0.001 & 0.0004 \\
\hline \multirow{2}{*}{ V-Human } & Mean \pm & $32.36 \pm$ & $13.97 \pm$ & $2.32 \pm$ & $0.053 \pm$ & $0.165 \pm$ & $16.03 \pm$ & $51.77 \pm$ & $3.43 \pm$ & $0.05 \pm$ & $0.09 \pm$ \\
& SE & 0.70 & 0.24 & 0.034 & 0.004 & 0.02 & 0.31 & 0.69 & 0.12 & 0.004 & 0.01 \\
\hline
\end{tabular}


Table (7): Significant differences of chemical contents of milk between different species

\begin{tabular}{|c|c|c|c|c|c|c|c|c|c|c|c||}
\hline \hline & & $\mathrm{Ca}$ & $\mathrm{P}$ & $\mathrm{Ca} / \mathrm{P}$ & $\mathrm{Fe}$ & $\mathrm{Zn}$ & $\mathrm{Na}$ & $\mathrm{K}$ & $\mathrm{Mg}$ & $\mathrm{Cu}$ & $\mathrm{Mn}$ \\
\hline \hline \multirow{5}{*}{ I-Camel } & Vs Buffalo & 0.0001 & 0.0001 & 0.003 & 0.0001 & 0.0001 & 0.004 & 0.04 & 0.0001 & 0.0001 & 0.0001 \\
\cline { 2 - 13 } & vs Cow & $\mathrm{NS}$ & 0.007 & 0.0001 & 0.0001 & 0.0001 & 0.0001 & 0.05 & 0.0001 & 0.0001 & 0.0001 \\
\cline { 2 - 13 } & vs Goat & 0.013 & 0.0001 & 0.0001 & 0.0001 & 0.0001 & 0.0006 & 0.0001 & 0.0001 & 0.0001 & $\mathrm{NS}$ \\
\cline { 2 - 12 } & Vs Human & 0.0001 & 0.0001 & 0.0001 & 0.0001 & 0.0001 & 0.0001 & 0.0001 & 0.0001 & 0.003 & 0.0001 \\
\hline II-Buffalo & vs Cow & 0.0001 & 0.0001 & 0.0001 & 0.008 & 0.0001 & $\mathrm{NS}$ & 0.0001 & 0.0001 & 0.0001 & 0.0001 \\
\cline { 2 - 12 } & vs Goat & 0.0001 & $\mathrm{NS}$ & 0.0001 & 0.0001 & 0.006 & $\mathrm{NS}$ & 0.0001 & 0.0001 & $\mathrm{NS}$ & 0.0001 \\
\cline { 2 - 11 } & Vs Human & 0.0001 & 0.0001 & 0.0001 & 0.0001 & 0.002 & 0.0001 & 0.0001 & 0.0001 & 0.04 & 0.0001 \\
\hline III-Cow & vs Goat & 0.0001 & 0.0001 & 0.0001 & $\mathrm{NS}$ & 0.03 & $\mathrm{NS}$ & 0.0001 & $\mathrm{NS}$ & 0.0001 & 0.0001 \\
\cline { 2 - 11 } & Vs Human & 0.0001 & 0.0001 & 0.0001 & $\mathrm{NS}$ & 0.0001 & 0.0001 & 0.0001 & 0.0001 & 0.0001 & 0.0001 \\
\hline IV-Goat & Vs Human & 0.0001 & 0.0001 & 0.0001 & $\mathrm{NS}$ & 0.0001 & 0.0001 & 0.0001 & 0.0001 & 0.02 & 0.0001 \\
\hline
\end{tabular}

Table (8): \% change of chemical contents of milk between and among different species.

\begin{tabular}{|c|c|c|c|c|c|c|c|c|c|c|c|}
\hline & & $\mathrm{Ca}$ & $\mathrm{P}$ & $\mathrm{Ca} / \mathrm{P}$ & $\mathrm{Fe}$ & $\mathrm{Zn}$ & $\mathrm{Na}$ & $\mathrm{K}$ & $\mathrm{Mg}$ & $\mathrm{Cu}$ & $\mathrm{Mn}$ \\
\hline \multirow[t]{4}{*}{ Camel } & Buffalo vs Camel & $46.54 *$ & $37.20 *$ & $7.31 *$ & |-40.49* & |-53.06* & |-10.78* & 6.94* & $341.15^{*}$ & $-34.17^{*}$ & $39.25 *$ \\
\hline & Cow vs Camel & 7.66 & $17.07 *$ & $-7.77 *$ & -69.78* & -25.54* & $\mid-14.13 *$ & $-5.95^{*}$ & $100.36^{*}$ & $-71.98^{*}$ & $-71.78^{*}$ \\
\hline & Goat vs Camel & $16.99 *$ & $35.72 *$ & $-13.60^{*}$ & $\mid-74.24 *$ & $\mid-36.97 *$ & $\mid-12.98 *$ & $28.87 *$ & 106.97* & $-35.67 *$ & 4.61 \\
\hline & Human vs Camel & $\mid-70.94 *$ & \begin{tabular}{|l|}
$-82.79 *$ \\
\end{tabular} & $69.23 *$ & $\mid-76.68 *$ & $\mid-67.65 *$ & $\mid-72.29 *$ & $-66.89 *$ & $-48.80 *$ & $-18.74 *$ & $582.11^{*}$ \\
\hline \multirow[t]{4}{*}{ Buffalo } & Camel & $-31.76^{*}$ & $-27.11^{*}$ & $-6.81 *$ & $68.05^{*}$ & 113.03* & $12.08 *$ & $-6.49 *$ & $-77.33^{*}$ & $51.91 *$ & $-28.18^{*}$ \\
\hline & Cow vs Buffalo & $-26.53 *$ & $-14.67 *$ & $-14.05^{*}$ & $-49.22 *$ & $58.63^{*}$ & -3.75 & $-12.06^{*}$ & $-54.58 *$ & $-57.44 *$ & $-79.73^{*}$ \\
\hline & Goat vs B & $-20.17 *$ & \begin{tabular}{|l|}
-1.07 \\
\end{tabular} & $-19.49 *$ & $-56.70 *$ & $34.27 *$ & -2.47 & $20.50 *$ & $-53.08 *$ & -2.28 & $-24.88^{*}$ \\
\hline & Hume & $-80.17 *$ & $-87.46^{*}$ & $57.70 *$ & $-60.82 *$ & -31.09* & $-68.94 *$ & $-69.04 *$ & $-88.39^{*}$ & $23.45^{*}$ & $389.86^{*}$ \\
\hline \multirow[t]{4}{*}{ Cow } & Came & -7.12 & -14.58* & $8.42 *$ & $230.95^{*}$ & $34.29 *$ & $16.45^{*}$ & $6.33^{*}$ & $-50.09^{*}$ & $256.95^{*}$ & $254.34 *$ \\
\hline & Buffa & $36.11 *$ & $17.19^{*}$ & $16.35^{*}$ & 96.94* & $-36.96 *$ & 3.90 & $13.71^{*}$ & $120.18^{*}$ & $134.97 *$ & $393.41 *$ \\
\hline & Goat & $8.66^{*}$ & $15.93 *$ & $-6.32 *$ & -14.73 & $-15.36 *$ & 1.34 & $37.03 *$ & 3.30 & $129.62 *$ & $270.66^{*}$ \\
\hline & Human vs Cow & $-73.01 *$ & $\mid-85.30 *$ & $83.49 *$ & -22.84 & $-56.56 *$ & $-67.73 *$ & $-64.79 *$ & -74.44* & 190.06* & $2316.97 *$ \\
\hline \multirow[t]{4}{*}{ Goat } & Camel vs Goa & $-14.52 *$ & $-26.32 *$ & $15.74 *$ & $288.13^{*}$ & $58.66^{*}$ & $14.92 *$ & $-22.40 *$ & $\mid-51.68 *$ & $55.45 *$ & -4.40 \\
\hline & Buffalo & $25.26^{*}$ & 1.09 & $24.21 *$ & $130.96^{*}$ & $-25.52 *$ & 2.53 & $-17.02 *$ & $113.14 *$ & 2.33 & $33.12 *$ \\
\hline & Cow & $-7.97 *$ & $-13.74 *$ & $6.75^{*}$ & 17.28 & $18.15^{*}$ & -1.32 & $-27.02 *$ & -3.20 & $-56.45^{*}$ & $-73.02 *$ \\
\hline & Hun & $-75.16^{*}$ & $-87.32 *$ & 95.88* & -9.51 & -48. & $-68.16^{*}$ & $-74.30 *$ & $-75.26^{*}$ & $26.32 *$ & $552.07 *$ \\
\hline \multirow[t]{4}{*}{ Human } & Came & $244.12 *$ & $481.20^{*}$ & $-40.91 *$ & $328.91 *$ & $209.13^{*}$ & $260.86^{*}$ & $201.98^{*}$ & $95.31^{*}$ & $23.06^{*}$ & $-85.34 *$ \\
\hline & Buffalo vs Human & $404.28 *$ & $697.39 *$ & $-36.59 *$ & $155.23^{*}$ & $45.11 *$ & $221.96^{*}$ & $222.95^{*}$ & $761.59^{*}$ & $-18.99^{*}$ & $-79.59^{*}$ \\
\hline & Cow vs Human & $270.49 *$ & $580.42 *$ & $-45.50 *$ & 29.60 & $130.19 *$ & 209.88 & $184.01 *$ & $291.31 *$ & $-65.52 *$ & $-95.86^{*}$ \\
\hline & Goat vs Human & $302.59 *$ & 688.82* & $-48.95^{*}$ & 10.51 & $94.84 *$ & $214.02 *$ & 289.16 & $304.23 *$ & $-20.84 *$ & $-84.66^{*}$ \\
\hline
\end{tabular}

*: Significant change 
Table (9): \% contribution of camel, buffalo, cow, goat and human milk intake with the recommended human daily dietary allowances, RDA (2002)

\begin{tabular}{|c|c|c|c|c|c|c|c|c|c|}
\hline & $\mathrm{Ca}$ & $\mathrm{P}$ & $\mathrm{Fe}$ & $\mathrm{Zn}$ & $\mathrm{Na}$ & $\mathrm{K}$ & $\mathrm{Mg}$ & $\mathrm{Cu}$ & $\mathrm{Mn}$ \\
\hline \multicolumn{10}{|l|}{ Children } \\
\hline Camel & 132.57 & 202.92 & 209.52 & 63.64 & 120.50 & 97.70 & 55.83 & 76.76 & 1096.98 \\
\hline Buffalo & 194.27 & 278.40 & 124.68 & 29.88 & 107.51 & 104.48 & 246.30 & 50.53 & 1527.50 \\
\hline Cow & 142.73 & 237.56 & 63.31 & 47.39 & 103.48 & 91.89 & 111.86 & 21.51 & 309.58 \\
\hline Goat & 155.10 & 275.41 & 53.98 & 40.11 & 104.86 & 125.91 & 115.55 & 49.38 & 1147.50 \\
\hline Human & 38.53 & 34.91 & 48.85 & 20.59 & 33.39 & 32.35 & 28.59 & 62.38 & 7482.55 \\
\hline \multicolumn{10}{|c|}{$7-12 \mathrm{M}$} \\
\hline Camel & 103.11 & 73.79 & 5.14 & 42.43 & 39.08 & 55.83 & 22.33 & 69.79 & 5.48 \\
\hline Buffalo & 151.10 & 101.24 & 3.06 & 19.92 & 34.87 & 59.71 & 98.52 & 45.94 & 7.64 \\
\hline Cow & 111.01 & 86.39 & 1.55 & 31.59 & 33.56 & 52.51 & 44.74 & 19.55 & 1.55 \\
\hline Goat & 120.63 & 100.15 & 1.33 & 26.74 & 34.01 & 71.95 & 46.22 & 44.89 & 5.74 \\
\hline Human & 29.96 & 12.70 & 1.20 & 13.73 & 10.83 & 18.49 & 11.43 & 56.71 & 37.41 \\
\hline \multicolumn{10}{|c|}{$18-50 y$} \\
\hline Camel & 27.84 & 28.99 & 7.07 & 11.57 & 9.64 & 8.31 & 3.99 & 17.06 & 1.43 \\
\hline Buffalo & 40.80 & 39.77 & 4.21 & 5.43 & 8.60 & 8.89 & 17.59 & 11.23 & 1.99 \\
\hline Cow & 29.97 & 33.94 & 2.14 & 8.62 & 8.28 & 7.82 & 7.99 & 4.78 & 0.40 \\
\hline Goat & 32.57 & 39.34 & 1.82 & 7.29 & 8.39 & 10.72 & 8.25 & 10.97 & 1.50 \\
\hline \multicolumn{10}{|c|}{$18-50 y$} \\
\hline Camel & 27.84 & 28.99 & 3.14 & 15.91 & 9.64 & 8.31 & 5.23 & 17.06 & 1.83 \\
\hline Buffalo & 40.80 & 39.77 & 1.87 & 7.47 & 8.60 & 8.89 & 23.09 & 11.23 & 2.55 \\
\hline Cow & 29.97 & 33.94 & 0.95 & 11.85 & 8.28 & 7.82 & 10.49 & 4.78 & 0.52 \\
\hline Goat & 32.57 & 39.34 & 0.81 & 10.03 & 8.39 & 10.72 & 10.83 & 10.97 & 1.91 \\
\hline
\end{tabular}

Table (10): Comparison of milks' mineral contents with other reported contents.

\begin{tabular}{|c|c|c|c|c|c|c|c|c|c|c|}
\hline & $\mathrm{Ca}$ & $\mathrm{P}$ & $\mathrm{Ca} / \mathrm{P}$ & $\mathrm{Fe}$ & $\mathrm{Zn}$ & $\mathrm{Na}$ & $\mathrm{K}$ & $\mathrm{Mg}$ & $\mathrm{Cu}$ & $\mathrm{Mn}$ \\
\hline \multicolumn{11}{|l|}{ Camel } \\
\hline$*$ & 111.36 & 81.17 & 1.37 & 0.23 & 0.51 & 57.84 & 156.32 & 6.70 & 0.061 & 0.013 \\
\hline 2 & 102.73 & 61.04 & 1.68 & 0.25 & 0.43 & 69.0 & 156.0 & 12.0 & & 0.0194 \\
\hline 3 & 118.16 & 76.85 & 1.54 & 0.13 & 0.49 & 58.09 & 170.37 & 7.44 & & 0.0083 \\
\hline \multicolumn{11}{|l|}{ Buffalo } \\
\hline$*$ & 163.19 & 111.36 & 1.47 & 0.135 & 0.24 & 51.61 & 167.18 & 29.56 & 0.04 & 0.01833 \\
\hline $4 \& 5$ & $\mid 169.0$ & 117.0 & 1.44 & 0.12 & 0.22 & 52.0 & 178.0 & 31.0 & $0.046 /-$ & $0.018 /-$ \\
\hline \multicolumn{11}{|l|}{ Cow } \\
\hline$*$ & $\mid 119.90$ & 95.03 & 1.26 & 0.07 & 0.38 & 49.67 & 147.02 & 13.42 & 0.017 & 0.0037 \\
\hline 4 & 119.0 & 93.0 & 1.28 & 0.05 & 0.38 & 49.0 & 152.0 & 13.0 & 0.011 & 0.004 \\
\hline 6 & & & & 0.045 & 0.39 & & & & 0.013 & 0.0022 \\
\hline 7 & & & & 0.02 & 0.4 & & & & 0.005 & 0.0021 \\
\hline 5 & 119.4 & 93.4 & 1.28 & 0.05 & 0.38 & 49 & 151.5 & 13.44 & 0.008 & 0.014 \\
\hline \multicolumn{11}{|l|}{ Goat } \\
\hline$*$ & 130.28 & 110.16 & 1.18 & 0.06 & 0.32 & 50.33 & 201.45 & 13.87 & 0.04 & 0.0138 \\
\hline 8 & 100 & 90 & 1.11 & 0.12 & 0.5 & 42.0 & 170 & 13.0 & 0.03 & - \\
\hline $4 \& 5 \& 9$ & 134.0 & 111.0 & 1.21 & 0.05 & 0.3 & 50.0 & 204.0 & 14.0 & 0.046 & 0.018 \\
\hline \multicolumn{11}{|l|}{ Human } \\
\hline$*$ & 32.36 & 13.97 & 2.32 & 0.053 & 0.165 & 16.03 & 51.77 & 3.43 & 0.05 & 0.09 \\
\hline 3 & 27.94 & 8.67 & 3.22 & 0.036 & 0.15 & 14.09 & 71.56 & 3.5 & & 0.0015 \\
\hline $4 \& 5$ & 32.0 & 14.0 & 2.29 & 0.03 & 0.17 & 17.0 & 51.0 & 3.0 & & \\
\hline 7 & & & & 0.026 & 0.215 & & & & 0.0314 & .0007 \\
\hline 10 & 28.0 & 15.0 & 1.87 & 0.04 & 0.166 & 15.0 & 58.0 & 3.0 & 0.035 & 0.0015 \\
\hline 11 & 28.0 & 14.0 & 2.0 & 0.036 & 0.24 & 14.0 & 45.0 & 3.0 & 0.035 & 0.004 \\
\hline
\end{tabular}

${ }^{1}$ Present study; ${ }^{2}$ Sawaya et al. $1984,{ }^{3}$ Gorban and Izzeldin, $1997 ;{ }^{4}$ Posati and Orr, 1976; ${ }^{5}$ Miller et al. 1999; ${ }^{6}$ Byron et al. 2005; ${ }^{7}$ Anderson, 1992; ${ }^{8}$ Holland et al.

1998; ${ }^{9}$ Alichanidis and Polychroniadou, 1996; ${ }^{10}$ Casey and Hambidge, 1983; ${ }^{11}$ Fomon, 1993 


\section{Discussion}

Results of human milk content agree with Posati and Orr (1976) and Fomon (1974 and 1993) except for carbohydrates and total solids. It disagrees with Renner (1983 and 1989) except for ash.

Result of Buffalo milk content agrees with Wong et al. (1988) except for protein, fat and carbohydrates and also agrees with Byron et al. (2005) except for fat. It seems thicker than cow's milk because it generally contained higher total solids than cow's milk $(+32.85 \%)$. In addition, its fat content is usually $+81.82 \%$ higher than cow's milk. Because of its high fat content, buffalo milk had considerably higher energy value than cow's milk.

Result of Goat milk content agrees with Posati and Orr. (1976) and Alichanidis and Polychroniadou. (1996). It disagree with Haenlein et al. (1992) where they found that main components of goat milk are similar to those of cow milk but differs as to particular physical and chemical properties (small size of fat globules, higher content of short and medium chain fatty acids). A significant minority of infants $(\geq 8 \%$ in 1 year old infants, Host et al., 1988) are allergic to one or more of the constituents of cow's milk so goats' milk could be equal or superior to cows' milk (Jenness 1980 and Hachelaf et al., 1993).

Result of Camel milk content disagrees with El-Bahay. (1962) and Knoess. (1976 and1977).

Result of Cow milk content agrees with Byron et al. (2005) and Renner (1983 and 1989).

Human milk contained the lowest protein content to meet infant's requirements and this reflects the slower growth of the infant relative to the other species. Also, the lower protein content of human milk lowers the milk buffering capacity and hence the osmotic stress to the kidney. The lower osmotic stress is important for kidney function, which has not fully developed in newborn or younger infant. The high content of protein for other milks may put a strain on an infant's immature kidney. In addition, the protein and fat in other milks are more difficult for an infant to digest and absorb than breast milk (Ogbu 2003 and Slusser and Powers 1997). Fat content of human milk is the most suitable source of energy. The average energy requirements of the newborn are about $100 \mathrm{Kcal} / \mathrm{kg} \mathrm{BW}$, while for adult $\approx$ $40.48 \mathrm{Kcal} / \mathrm{kg} \mathrm{BW}$, i.e. newborn infants require 2-3 times as adults, which is explained by the newborn's high basal metabolism. Human milk is characterized by higher carbohydrates content; usually the disaccharide lactose, which have a low osmotic value per unit of weight, which is relevant to the infant's water balance. Infants has a relatively high water requirements because their relatively large body surface and hence a high evaporation. Lactose decomposes in the gastro-intestinal canal at a relatively slow rate, consequently, part of it reaches the terminal ileum and colon unsplit and could contribute there to the formation of the socalled bifidus flora (Hamosh 1992 and Lawrence and Lawrence 2005). The fat globule in goat's milk does not cluster together due to absence of agglutinin, which makes goat's milk easier for an infant to digest. Also goat's milk does not contain many of the allergens found in cows' or other milks, and yet goat's milk is unsuitable for infants as it can cause intestinal irritation and anaemia (Desjeux 1993; Bindal and Wadhwa 1993; Slusser and Powers 1997 and Lawrence and Lawrence 2005).

Calcium is very important to human being. It is needed for tissue and bone development and adequate calcium intake is important for maintenance of bone health and may reduce risk of osteoporosis. Calcium can be obtained from foods naturally rich in calcium such as milk and dairy foods. Milk and other dairy foods are the major source of calcium in Egypt. It helps nerves conduct messages; muscle contractions; blood clotting; signaling the heart muscle (WHO/FAO, 1996). Calcium content of camel milk disagrees with Sawaya et al. (1984) and Gorban and 


\section{Ghada Z A Soliman}

Izzeldin (1997). Calcium content of buffalo, cow, goat and human milk agrees with Posati and Orr (1976), Miller et al. (1999) and Alichanidis and Polychroniadou (1996). Calcium content of goat milk disagrees with Holland et al. (1998). Calcium content of human disagrees with Gorban and Izzeldin (1997); Casey and Hambidge (1983) and Fomon (1993). They provide: infants/ serving $\leq 6$ months with 194.27, $155.1 \%$ of their RDA (DRI); infants $\leq 1$ year with $151.1,120.63$ of their RDA (DRI) respectively, while human milk provide infants with $38.53,29.96 \%$ of their RDA (DRI) respectively. Buffalo and goat milk contain 3-4.5 times of the human milk content. They provide adult (male or female) with $27.84-40.8 \%$ of their RDA (DRI).

Phosphorous is important in maintaining acid-base balance; protein and energy metabolism; maintains structure of cell membranes. Phosphorous works with calcium to develop and maintain strong bones and teeth and enhances the use of other nutrients (WHO/FAO, 1996). Phosphorous content of camel milk disagree with Sawaya et al. (1984) and Gorban and Izzeldin (1997). Phosphorous content of buffalo and cow milk agrees with Posati and Orr (1976) and Miller et al. (1999). Phosphorous content of goat milk agrees with Posati and Orr (1976), Miller et al. (1999) and Alichanidis and Polychroniadou (1996) and disagrees with Holland et al. (1998). Phosphorous content of human milk agrees with Posati and Orr (1976), Miller et al. (1999), Casey and Hambidge (1983) and Fomon (1993) and disagrees with Gorban and Izzeldin (1997).

Human milk contain the highest, significant Ca: $\mathrm{P}$ ratio, 2.32: 1. This higher ratio prevents the development of hypocalcæmia and secondary hyperparathyroidism in the newborn. In particular in the first months of life, the infant may have difficulties in phosphate excretion, which may lead to hyperphosphatæmia and ultimately to hypocalcæmia and tetany (Fomon, 1993). $\mathrm{Ca}$ : $\mathrm{P}$ ratio of camel milk disagrees with Sawaya et al. (1984) and Gorban and Izzeldin (1997). Ca: P ratio of buffalo and cow milk agrees with Posati and Orr (1976) and Miller et al. (1999). Ca: P ratio of goat milk agrees with Posati and Orr (1976) and Miller et al. (1999) and Alichanidis and Polychroniadou (1996) and disagrees with Holland et al. (1998). Ca: P ratio of human milk agrees with Posati and Orr (1976) and Miller et al. (1999) and disagrees with Gorban and Izzeldin (1997) and Casey and Hambidge (1983). In this study buffalo and goat milk provide a great amount of phosphorus. They provide: infants/ serving $\leq 6$ months with $278.40,275.41 \%$ of their RDA (DRI); infants $\leq 1$ year with 101.24, 100.15 of their RDA (DRI) respectively, while human milk provide infants with $34.91,12.70 \%$ of their RDA (DRI) respectively. Buffalo and goat milk contain 7 times of human milk content. They provide adult (male or female) with $\approx 39.5$ $\%$ of their RDA (DRI).

Iron is vital components of blood hæmoglobin required for oxygen transportation and enzyme systems and is necessary for red blood cell formation and function, and for brain function (WHO/FAO, 1996). Iron content of camel milk agrees with Sawaya et al .(1984) and disagrees with Gorban and Izzeldin (1997) . Iron content of buffalo and cow milk disagrees with posati and Orr (1976) and Miller et al. (1999), Byron et al. (2005) and Anderson (1992) Iron content of goat milk disagrees with Posati and Orr (1976) and Miller et al. (1999), Alichanidis and Polychroniadou (1996) and Holland et al. (1998) . Iron content of human milk disagrees with posati and Orr ( 1976) and Miller et al .( 1999). Gorban and Izzeldin (1997) and Casey and Hambidge (1983). In this study camel then buffalo milk provide a great amount of iron. They provide: infants/ serving $\leq 6$ months with 209.52, $124.68 \%$ of their RDA (DRI); infants $\leq 1$ year with $5.14,3.06$ of their RDA (DRI) respectively, while human milk provide infants with $48.85,1.20 \%$ of their RDA (DRI) respectively. Camel and Buffalo milk contain 4-2.5 times of the human milk content. They provide adult male with 7.07, $4.21 \%$; adult female with $3.14,1.87 \%$ of their RDA (DRI). Surprisingly milk provides infants $\leq 6$ months with a lot of Fe. Milk provide $>100 \%$ of their need from 
camel, buffalo, cow and goat while human milk provide $\approx 50 \%$. This ratio decreases with age where it reaches $1.33-5.14 \%$ with infants $\leq 1$ year. Even more, milk provide adult male with double (twice) as female although the ratio is small (male: 1.82$7.07 \%$ and female: $0.81-3.14 \%$ ).

Zinc is essential part of more than 200 enzymes involved in digestion, metabolism, reproduction and wound healing (WHO/ FAO 1996). Zinc content of camel milk disagrees with Sawaya et al. (1984) and agrees with Gorban and Izzeldin (1997). Zinc content of buffalo and cow milk agrees with Posati and Orr (1976) and Miller et al. (1999), Byron et al. (2005) and Anderson (1992). Zinc content of goat milk agrees with Posati and Orr (1976) and Miller et al. (1999) and Alichanidis and Polychroniadou (1996) and disagrees with Holland et al. (1998). Zinc content of human milk agrees with Posati and Orr (1976) and Miller et al. (1999) and Casey and Hambidge (1983) and disagrees with Gorban and Izzeldin (1997) and Anderson (1992). In most species, including human being, zinc concentration falls as lactation advances (Cassey et al. 1989). This could probably be due to depletion of maternal zinc stored (Krebs et al. 1985). They (camel, buffalo, cow and goat milk) provide: infants $\leq 6$ months with 29.88$63.64 \%$ of their RDA (DRI); infants $\leq 1$ year with 19.92-42.43\% of their RDA (DRI), while human milk provide infants with $20.59,13.73 \%$ of their RDA (DRI) respectively. Buffalo and goat milk contain 7 times human milk content of $\mathrm{Zn}$. Buffalo and goat milk provide adult (male or female) with $\approx 39.5 \%$ of their RDA (DRI).

Sodium is important in maintaining water balance; maintaining acid-base balance; muscle contraction; nerve contraction (WHO/FAO 1996). Sodium content of camel milk disagrees with Sawaya et al. (1984) and agrees with Gorban and Izzeldin (1997). Sodium content of buffalo, goat and cow milk agrees with Posati and Orr (1976), Miller et al. (1999) and Alichanidis and Polychroniadou (1996). Sodium content of goat milk disagree with Holland et al. (1998). Sodium content of human milk agrees with Posati and Orr (1976) and Miller et al. (1999) and Casey and Hambidge (1983) and disagrees with Gorban and Izzeldin (1997), Desjeux (1993) and Fomon (1993). They (camel, buffalo, cow and goat milk) provide: infants $\leq 6$ months with $103.48-120.5 \%$ of their RDA (DRI); infants $\leq 1$ year with 33.56$39.08 \%$ of their RDA (DRI), while human milk provide infants with $33.39,10.83 \%$ of their RDA (DRI) respectively. Camel, buffalo, cow and goat milk contain 3-4 times human milk content of Na. Camel, buffalo, cow and goat milk provide adult with $\approx 9.0 \%$ of their RDA (DRI).

Potassium is important in nerve conduction; maintaining water and fluid balance; maintain acid-base balance; muscle contraction. It regulates heartbeat (WHO/FAO, 1996). Potassium content of camel milk agrees with Sawaya et al. (1984) and disagrees with Gorban and Izzeldin (1997). Potassium content of buffalo and cow milk disagrees with Posati and Orr (1976) and Miller et al. (1999). Potassium content of goat milk agrees with Posati and Orr (1976) and Miller et al. (1999) and Alichanidis and Polychroniadou (1996) and disagrees with Holland et al. (1998). Potassium content of human milk agrees with Posati and Orr (1976) and Miller et al. (1999) and disagrees with Gorban and Izzeldin (1997), Casey and Hambidge (1983), and Fomon (1993). In this study goat then buffalo milk provide a great amount of potassium. They provide: infants $\leq 6$ months with $125.91,104.48 \%$ of their RDA (DRI); infants $\leq 1$ year with $71.95,59.71$ of their RDA (DRI) respectively, while human milk provide infants with $32.35,18.49 \%$ of their RDA (DRI) respectively (table 9). Buffalo and goat milk contain 4-3 times of the human milk content. They provide adult (male or female) with 10.72-8.89 \% of their RDA (DRI).

Magnesium activates 100 enzymes and play role in over 300 enzyme reactions in the body, many of which are directly related to cardiovascular health and helps nerves and muscles function. Manganese is important in protein metabolism; bone and teeth formation (WHO/FAO 1996). 
Magnesium content of camel milk disagrees with Sawaya et al. (1984) and Gorban and Izzeldin (1997). Magnesium content of buffalo, cow and goat milk agrees with Posati and Orr (1976) and Miller et al. (1999), Alichanidis and Polychroniadou (1996) and Holland et al. (1998). Magnesium content of human milk agrees with Gorban and Izzeldin (1997) and disagrees with Posati and Orr (1976) and Miller et al. (1999), Casey and Hambidge (1983), and Fomon (1993). In this study buffalo then goat milk provide a great amount of magnesium. They provide: infants $\leq 6$ months with $246.3,115.55 \%$ of their RDA (DRI); infants $\leq 1$ year with 98.52, 46.22 of their RDA (DRI) respectively, while human milk provide infants with $28.59,11.43 \%$ of their RDA (DRI) respectively. Buffalo, cow and goat milk contain 8.6-4 times human milk content of $\mathrm{Mg}$. They provide adult male with $17.59,7.99,8.25 \%$, and adult female with $23.09,10.49$, and $10.83 \%$ of their RDA (DRI).

Copper is important in normal redblood cell formation, Connective tissue formation, acts as a catalyst to store and release iron to help form hæmoglobin. Contributes to central nervous system function (WHO/FAO 1996). Copper content of camel, buffalo, cow, goat and human milk disagrees with all mentioned reports here (2-11). In this study camel and human milk provide a great amount of copper. They provide: infants $\leq 6$ months with $76.76,62.38 \%$ of their RDA (DRI); infants $\leq 1$ year with $69.76,56.71 \%$ of their RDA (DRI) respectively. Buffalo, cow and goat milk contain 3-1.2 times human milk content of $\mathrm{Cu}$. Buffalo, cow and goat milk provide adult male or female with $10.97 \%$.

Manganese is an essential trace mineral that plays important roles in reproductive hormone production, urea excretion, immunity, and joint/leg development (WHO/FAO, 1996). Magnesium content of camel milk disagrees with Sawaya et al. (1984) and Gorban and Izzeldin (1997). Magnesium content of buffalo milk agrees with Posati and Orr (1976) and Miller et al. (1999). Magnesium content of cow agrees with Posati and Orr
(1976) and disagrees with Miller et al. (1999). Byron et al. (2005) and Anderson (1992). Magnesium content of goat milk disagrees with Posati and Orr (1976), Miller et al. (1999) and Alichanidis and Polychroniadou (1996). Magnesium content of human milk disagrees with Gorban and Izzeldin (1997), Anderson (1992), Casey and Hambidge (1983), and Fomon (1993). Human milk contained $\approx 6.5$ times camel and cow milk and 24.14 times as goat milk. In this study human milk provide a great amount of Mn. All tested milks provide: infants $\leq 6$ months with more than they need, > of their RDA (DRI); Human milk provide infants $\leq 1$ year with 37.41 of their RDA (DRI) respectively, while remaining milk provide infants with 1.55-7.64 \% of their RDA (DRI) respectively and provide adults (male and female with $0.4-2.55 \%$ of their RDA (DRI).

The mineral content of human, cow and goat disagree with Belewu and Aiyegbusi (2002). The variation in mineral concentration of camel, buffalo, cow and goat with other cited reports is due probably to the breed, period of lactation and dietary content (ICAR, 1981). Age of mothers, number of previous children, period and/or season of lactation and dietary content and type of delivered baby (full or preterm) may lead to the variation in mineral concentration of human milk (Rodriguez et al,. $2000 \& 2002$ and Aquilio et al. 1996). The reported levels of $\mathrm{Zn}, \mathrm{Cu}, \mathrm{Fe}, \mathrm{Mg}$ and $\mathrm{Mn}$ in human milk vary widely. The range of reported mean values $(\mathrm{mg} / 100 \mathrm{~g})$ for $\mathrm{Fe}$ is 0.02-0.073; for $\mathrm{Cu} 0.024-0.05$; for $\mathrm{Zn}$ 0.116-0.383; for Mg 2.6-3.5 (Fransson and Lönnerdal 1982, 1983 and Al-Awadi and Srkumar, 2000). Our levels in this study of Fe 0.053; Cu 0.05; Zn 0.165 and $\mathrm{Mg} 3.43$ lie within these ranges.

\section{CONCLUSION}

Mammals as camel, buffalos, cow, goat and human being produce milk, but the composition of milk for each species varies widely and other kinds of milk often vary different from human breast milk. From the viewpoint of human nutrition, despite goat's milk is easier for an infant to digest and it does not contain many of the 
allergens found in cow's milk and that other milk may have higher mineral content than human milk but still cannot replace human milk in young children but could complement it.

\section{References}

1. Al-Awadi FM, Srikumar TS. (2000): Trace-element status in milk and plasma of Kuwaiti and non-Kuwaiti lactating mothers. Nutrition. 16: 1069-1073.

2. Alichanidis E, Polychroniadou A. (1996): Special features of dairy products from ewe and goat milk from the physicochemical and organoleptic point of view. In: Proceedings, Production and Utilization of Ewe and Goat Milk, Crete, Greece, Oct. 1921, 1995, International Dairy Federation Publ., Brussels, Belgium, p. 21-43.

3. Ambrosoli R, Stasio L di and Mazzocco P. (1998): Content of alpha-s-1-casein and coagulation properties in goat milk. J. Dairy Sci,: 71: 24-28.

4. Anderson R R. (1992): Comparison of Trace elements in milk of four species. $\mathbf{J}$ Dairy Sci.; 75: 3050-3055.

5. Aquilio E, Spagnoli R, Seri S, Bottone G and Spennati G. (1996): Trace element content in human milk during lactation of preterm newborns. Biol Trace Elem Res.; 51(1): 63-70.

6. AOAC (1981): "Official method of analysis of the Association of Official Analytical Chemists. Published by the association of official analytical chemists, Inc., Arlington, USA.

7. AOAC (1990): "Official method of analysis of the Association of Official Analytical Chemists. Published by the association of official analytical chemists, Inc., Arlington, USA.

8. Bates C J and Prentice A. (1994): Breast milk as a source of vitamins, essential minerals and trace elements. Pharmacol Ther.; 62(1-2): 193-220.

9. Belewu M A and Aiyegbusi O F. (2002): Comparison of the Mineral Content and Apparent Biological Value of Milk from Human, Cow and Goat. The Journal of Food Technology in Africa, Vol. 7, JanMar, pp. 9-11.

10. Bindal M P and Wadhwa B K. (1993): Compositional differences between goat milk fat and that of cows and buffaloes. Small Rumin. Res. 12:79-88.
11. Byron H W, Arnold $H J$ and John A A. (2005). Fundamentals of dairy chemistry. 2 ${ }^{n d}$ Ed. CBS, BS publishers and distributors.

12. Casey $C$ E and Hambidge K M. (1983): Nutritional aspects of human lactation. In: MC Neville, MR Neifert (eds.), Lactation: physiology, nutrition and breatfeeding. New York: Plenum, p: 203-204.

13. Charan C. (1994): Asian Buffalo Association Congress 63, Sasaki.

14. Daves G K and Mertz W. (1986): Copper. Page 301. In: Trace elements in human and animal nutrition. Vol. 1. $5^{\text {th }}$ ED. W. Mertz ed. Academic Press, New York, N Y.

15. Desjeux J F. 1993. Nutritional value of goat milk. Lait 73:573-580.

16. DRIs, Dietary Reference Intakes. (2002): Recommended Intakes for Individuals. Macronutrients, Elements. Food and Nutrition Board, Institute of Medicine, National Academies.

17. Elamin F M and Wilcox C J. (1992). Milk Composition of Majaheim Camelsi. J. Dairy Sci. 75: 3155-3157.

18. El-Bahay G M. (1962): Normal contents of Egyptian camel milk. Vet. Med. J,; 8: 717.

19. Fomon S J. (1974): Nutritional requirements in relation to growth. Monatsschr Kinderheilkd,; 122: suppl: 236-239.

20. Fomon S J. (1993): Nutrition of normal infants. Mosby, St. Louis.

21. Fransson G-B and Lönnerdal Bo. (1982): Zinc, copper, calcium and magnesium in human milk. J. Pediat., 101: 504-508.

22. Fransson G-B and Lönnerdal Bo (1983): Distribution of trace elements and minerals in human and cow's milk. Pediatr Res.; 17(11): 912-915.

23. Gorban A M S and Izzeldin O M (1997): Mineral content of camel milk and colostrum, J. Dairy Sci. 64: 471-474.

24. Hachelaf W, Boukhrelda M, Benbouabdellah M, Coquin P, Desjeux J F, Boudraa G and Touhami M. (1993): Comparative digestibility of goat versus cow's milk fats in children with digestive malnutrition. Lait 73:593-599.

25. Haenlein G F W. (1992): Role of goat meat and milk in human nutrition. Proceedings Vth International Conference on Goats, New Delhi, India, March 1-8, ICAR Publ., New Delhi, 2 (II): 575-580.

26. Hamosh M. (1992): Human milk composition and the function in the infant. Seminars in Pediatric Gastroenterology and Nutrition.; 3(3): 4-8. 
27. Holland B, Welch A A, Unwin D H, Buss D H, Pall A A, and Southgate D A T. (1998): McCance and Winddowson's, The composition of foods. $5^{\text {th }}$ Ed., The Royal Society of Chemistry, Ministries of Agriculture, Fisheries and Food. MAFF.

28. Host A, Husby $\mathbf{S}$ and Osterballe $\boldsymbol{O}$. (1988). A prospective study of cow's milk allergy in exclusively breast-fed infants. Acta Paediatr. Scand. 77: 663-670.

29. ICAR, Indian Council for Agricultural Research. (1981): A Handbook of Animal Husbandry. Edited by SHRI. P.J. Joseph for the Indian Council of Agricultural Research, New Delhi. Pg. 99.

30. Jenness R. (1980): Composition and characteristics of goat milk: Review 19681979. J. Dairy Sci. 63:1605-1630.

31. Krebs N F and Hambique K M. (1985). The effects of dietary $\mathrm{Zn}$ supplement during lactation. Amr. J. Clin. Nutrition; 41: 560570.

32. Knoess K H. (1976): Assignment reports on animal production in the Middle Awash Valley.; FAO, Rome,

33. Knoess K H. (1997): The camel as a meat and milk animal. World Anim. Rev,; 22392245.

34. Lawrence $\mathbf{R} A$ and Lawrence $R \mathbf{M}$. (2005): Breastfeeding, A Guide for the Medical Profession, 6th Ed. Elsevier Mosby, Phila.; p73-86, p105-214.

35. Lothe $\mathbf{L}$, Lindberg $\mathbf{T}$ and Jacobson $\mathbf{I}$. (1982). Cow's milk formula as a cause of infantile colic. A double-blind study Pediatrics, 70: 7-10.

36. Miller GD,Jarvis J K, and McBean LD.( 1999) : Handbook of Dairy foods and Nutrition. 2 ndEd. Edited by lra Wolinsky.

37. Park Y W. (1991): Relative buffering capacity of goat milk, cow milk, soy-based infant formulae and commercial nonprescription antacid drugs. J. Dairy Sci. 74:3326-3333.

38. Ogbu ISI (2003) : The biochemical composition of the breast milk of Nigerian mothers. Mary Slessor J of Medicine Vol.3 (1): 1-4.

39. Posati L P and Orr M L. (1976) : Composition of Foods. Dairy and Egg Products. USDA-ARS. Consumer and Food
Economics Inst., Agr. Handbook, Washington D.C; No.8-1,77-109

40. RDAs. (2002): Recommended Dietary Allowances. Recommended Intakes for Individuals. Macronutrients, Elements. Food and Nutrition Board, Institute of Medicine, National Academies

41. Renner E. (1983): Milk and dairy products in human nutrition. Volkswirtschaftlicher Verlag, Munich, Germany.

42. Renner E. (1989): Micronutrients in milk and milk-based products. Elsevier Applied Science, London.

43. Rennert $O$ M and Chan W-Y. (1984): Metabolism of Trace Metals in Man, Vol. I. CRC Press, p. 71.

44. Rodriguez Rodriguez EM, Sanz Alaejos $M$ and Diaz Romero C. (2000): Concentrations of iron, copper and zinc in human milk and powdered infant formula. Int J Food Sci Nutri. 51(5): 373-380.

45. Rodriguez Rodriguez EM, Sanz Alaejos $M$ and Diaz Romero C. (2002): Concentrations of calcium, magnesium, sodium and potassium in human milk and infant formulas. Arch Latinoam Nutr., 52(4): 406-412.

46. Sawaya $W$ N, Khalil A, Al-Shalhat $A$ and Al-Mohammad H. (1984): Chemical composition and nutritional qualify of camel milk. J. Food Sci. 49:744-74

47. Slusser W and Powers N G (1997): Breastfeeding update 1: immunology, nutrition, and advocacy. Pediatrics in Review.; 18(4): 111-119.

48. Steel R G D and Torrie J H. (1969): Principles and procedures of statistics McGraw-Hill Co. New York.

49. Whitehead R G. (1995): For how long is exclusive breast feeding adequate to satisfy the dietary energy needs of the average young baby? Pediatr. Res. 37: 239-245.

50. Wong $\mathbf{N} \mathbf{P}$, Jenness $\mathbf{R}$, Keeney $\mathbf{M}$ and Marth E H. (1988): Fundamentals of Dairy Chemistry. Van Nostrand Reinhold Company, New York, NY.

51. WHO/FAO report. (1996): Trace elements in Human nutrition and health.

52. Yagil R, Saran A and Etzion Z. (1984): Camels' milk: for drinking only? Comp Biochem Physiol A.; 78(2): 263-266. 


\title{
مقارنه بين المحتوى الكيميائي ومحتوى المعادن في ألبان الإنسان

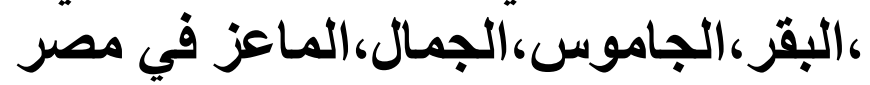

\author{
غاده ز غلول عباس سليمان \\ زميل كيمياء حيوية قسم الكيمياء الحيوية المعهد القومى للتغذية ـألقاهره
}

يعتبر اللبن غذاء مهم ومصدر ا جيدا للكالسيوم ان المقارنهـ بين أنواع اللبن في مصر

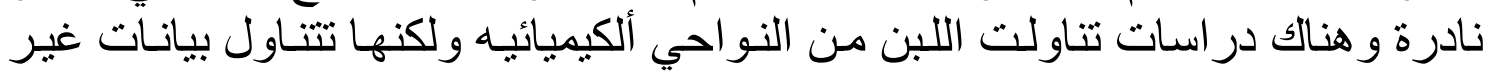

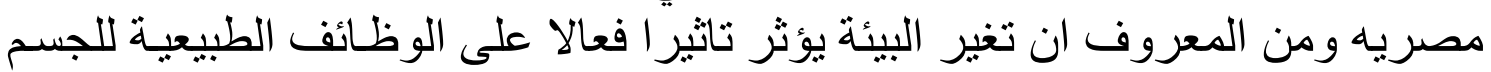

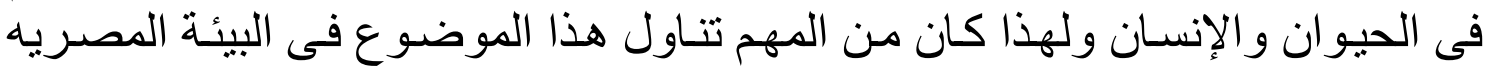

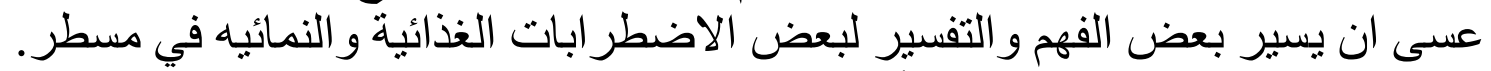

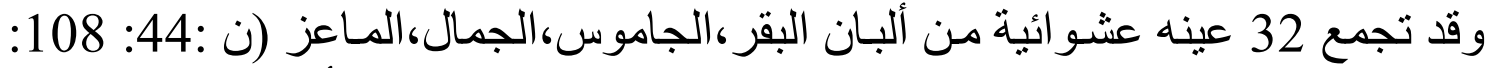

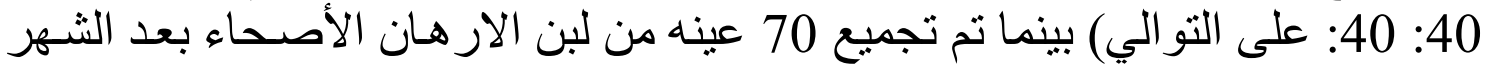

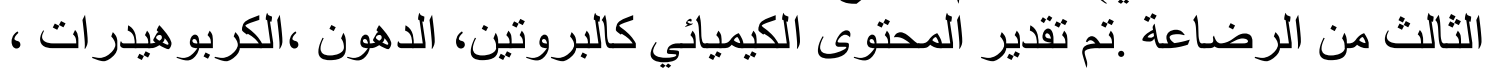

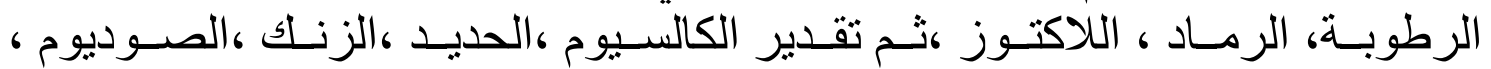

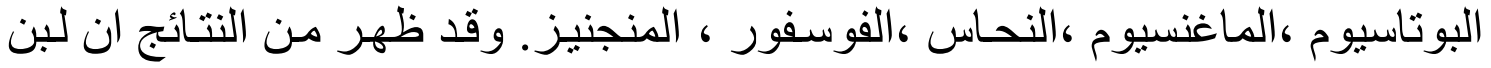

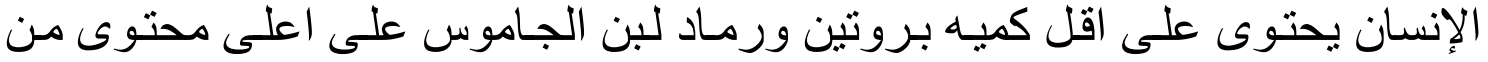

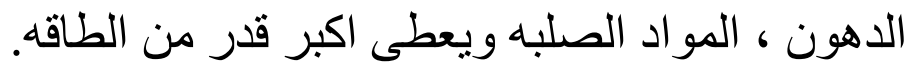

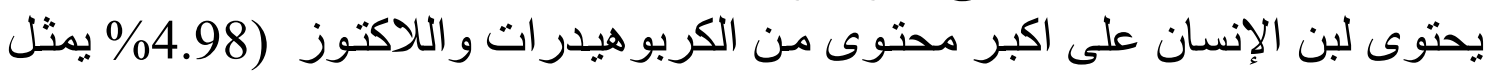

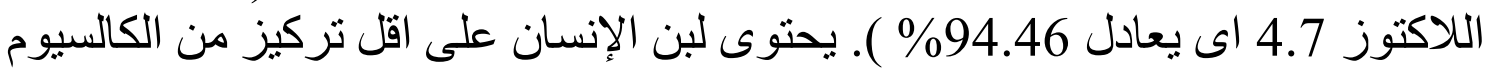

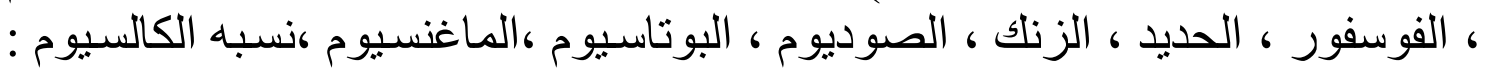

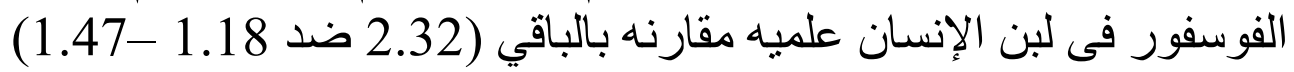

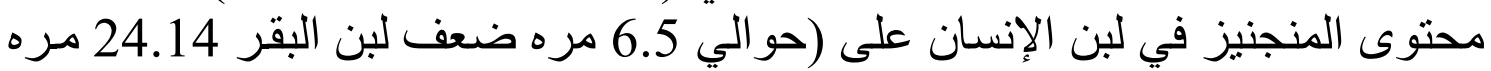

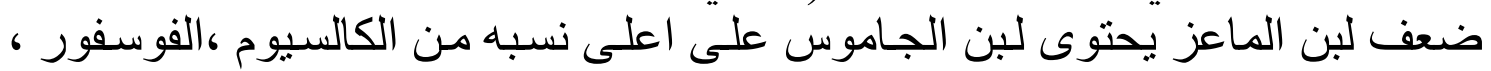

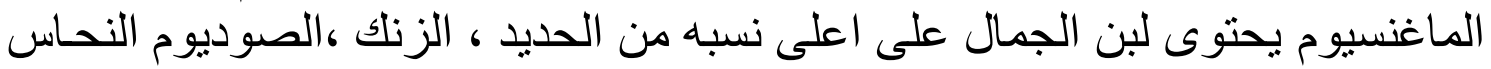

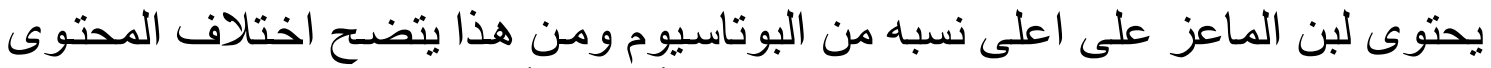

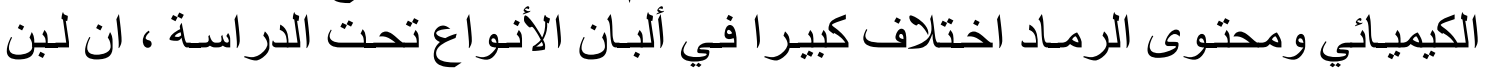
الجاموس، الجمال ،الماعز ،البقر ،لا يحل محل لبن الإنسان ولكن الإن يكمله. 